\title{
Hiperoxia por dos horas produce daño morfológico cerebral luego de asfixia neonatal experimental
}

Melva Benavides, Roberto Shimabuku, Arturo Ota, Sonia Pereyra, Carlos Delgado, Víctor Sánchez, Graciela Nakachi, Pablo Velásquez, Flor Cruz

Instituto Nacional de Salud del Niño

Objetivos: Determinar el efecto de una exposición por 2 horas de hiperoxia al 40\% y 100\% sobre la morfología cerebral, en un modelo experimental de asfixia neonatal.

Diseño: Experimental.

Institución: Instituto Nacional de Salud del Niño.

Material biológico: Muestra constituida por 120 ratas albinas Holtzman de 1 semana de nacidas, asignadas aleatoriamente.

Intervenciones: Todos los animales (a excepción del grupo control) fueron sometidos previamente a asfixia experimental (O2 al 8\%); luego, fueron expuestos a $\mathrm{O} 2 \mathrm{al} 100 \%$ y $\mathrm{O} 2 \mathrm{al} 40 \%$, ambas por 2 horas, a $\mathrm{O} 2 \mathrm{al} 21 \%$ y grupo control.

Principales medidas de resultados: Presencia de daño cerebral macroscópico, peso cerebral, porcentaje de daño cerebral microscópico.

Resultados: El modelo experimental de asfixia neonatal en ratas produjo disminución del peso cerebral a los 7 días en los diferentes grupos de experimentación. El daño cerebral microscópico fue con mayor frecuencia en el grupo sometido a hipoxia experimental y que recibió $02100 \%$ por 2 horas y con menor frecuencia el que recibió 02 al $40 \%$ (60\% vs. $43,3 \%)$, diferencia estadísticamente significativa $(p<0,001)$. El grupo que recibió $02100 \%$ tuvo mayor porcentaje de área cerebral con daño microscópico (18,3\%).

Conclusiones: El modelo experimental de asfixia neonatal en ratas generó menor peso cerebral y el daño histológico fue más evidente en el grupo con hiperoxia al 100\%.

Palabras clave: Asfixia experimental, hiperoxia, daño cerebral.

\section{Caracterización molecular de la inmunidad humoral de la paragonimiosis experimental}

Alina Huiza, Carlos Sevilla, William Cornejo, Marcos Ñavincopa, Pilar Alva, Eva Casas, Amanda Chávez, Abraham Cáceres, Abelardo Tejada, Juan Medina, Diego Moreno, Christina Gallardo, Jorge Maximiliano, Rosendo Ccenta, Pilar Díaz, José Gonzales, Jaime Soria, Rubén Vásquez

Instituto de Medicina Tropical Daniel A. Carrión, Facultad de Medicina, UNMSM

Objetivos: Caracterizar las moléculas de respuesta humoral anti Paragonimus mexicanus/P. peruvianus para diferenciar la infección en fase aguda y fase crónica, mediante la evaluación de la dinámica de la respuesta inmune.

Diseño: Observacional, longitudinal.

Institución: Instituto de Medicina Tropical Daniel A. Carrión, Facultad de Medicina, UNMSM

Material biológico: Animales de experimentación.

Principales medidas de resultados: Determinación de lgG e IgM anti Paragonimus.

Resultados: Existió diferencia significativa de medias de la DO entre los grupos crónico y no infectados para los anticuerpos IgG anti ES de Paragonimus. Los anticuerpos IgM anti Paragonimus tuvieron una ligera elevación entre los días 7 a 20 post inoculación (pi), y una tendencia hacia la disminución entre los días 40 y 60 pi.

Conclusiones: La dinámica de producción de anticuerpos lgG e lgM anti Paragonimus mediante Elisa indirecto, diferenció la fase aguda de la fase crónica, en los gatos inoculados.

Palabras clave: Paragonimus, Elisa IgG, Elisa IgM, inmunidad humoral 